Original article

\title{
Gastroesophageal reflux disease and Barrett's esophagus after laparoscopic sleeve gastrectomy: a possible, underestimated long-term complication
}

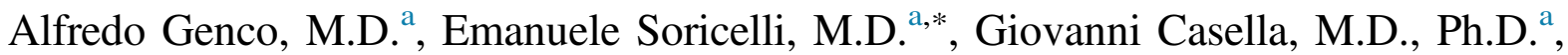 \\ Roberta Maselli, M.D. ${ }^{a}$, Lidia Castagneto-Gissey, M.D. ${ }^{a}$, Nicola Di Lorenzo, M.D. ${ }^{\text {, }}$, \\ Nicola Basso, M.D. ${ }^{\mathrm{a}}$ \\ ${ }^{a}$ Department of Surgical Sciences, Policlinico Umberto I, Sapienza University of Rome, Rome, Italy \\ ${ }^{b}$ Department of Experimental Medicine and Surgery, Tor Vergata University of Rome, Rome, Italy \\ Received July 28, 2016; revised October 11, 2016; accepted November 11, 2016
}

Abstract

\begin{abstract}
Background: Morbidly obese patients are affected by gastroesophageal reflux disease (GERD) and hiatal hernia $(\mathrm{HH})$ more frequently than lean patients. Because of conflicting results, the indication to sleeve gastrectomy (SG) in patients with GERD is still debated.

Objectives: To evaluate the incidence of GERD on the basis of clinical, endoscopic, and histologic data in patients undergoing SG.

Settings: University hospital, Rome, Italy.

Methods: From July 2007 to January 2010, 162 patients underwent primary SG. Preoperatively all patients underwent visual analogue scale (VAS) evaluation of GERD symptoms, proton pump inhibitors (PPIs) consumption recording, and esophagogastroduodenoscopy (EGD). Stomach resection started $6 \mathrm{~cm}$ from pylorus on a 48Fr bougie. Staple line was reinforced by an oversewing suture. A postoperative clinical control with VAS evaluation, PPI consumption, and EGD was proposed to all patients. Three patients were excluded because of the occurrence of major postoperative complications. Results: A total of 110 patients accepted to take part in the study (follow-up rate: 69.1\%). At a mean 58 months of follow-up, incidence of GERD symptoms, VAS mean score, and PPI intake significantly increased compared with preoperative values $(68.1 \%$ versus $33.6 \%: P<.0001 ; 3$ versus $1.8: P=.018 ; 57.2 \%$ versus $19.1 \%: P<.0001)$ At $\mathrm{EGD}$, an upward migration of the " $\mathrm{Z}$ " line and a biliary-like esophageal reflux was found in $73.6 \%$ and $74.5 \%$ of cases, respectively. A significant increase in the incidence and in the severity of erosive esophagitis (EE) was evidenced, whereas nondysplastic Barrett's esophagus (BE) was newly diagnosed in 19 patients (17.2\%). No significant correlations were found between GERD symptoms and endoscopic findings.

Conclusion: In the present series the incidence of EE and of BE in SG patients was considerably higher than that reported in the current literature, and it was not related to GERD symptoms. Endoscopic surveillance after SG should be advocated irrespective of the presence of GERD symptoms. (Surg Obes Relat Dis 2017;13:568-574.) (C) 2017 American Society for Metabolic and Bariatric Surgery. All rights reserved.
\end{abstract}

\footnotetext{
${ }^{*}$ Correspondence: Emanuele Soricelli, M.D., Department of Surgical Sciences, Policlinico Umberto I, Sapienza University of Rome, Viale Regina Elena 324, 00161, Rome, Italy.

E-mail: emanuele.soricelli@gmail.com
}

Morbidly obese patients are affected by gastroesophageal reflux disease (GERD) and hiatal hernia (HH) more frequently than lean patients [1]. In turn, GERD and $\mathrm{HH}$ 
are associated with an increased incidence of Barrett's esophagus (BE). In particular the duration and the degree of reflux symptoms, as well as the presence of $\mathrm{HH}$, appear to be positively related to the prevalence of $\mathrm{BE}$ [2]. Furthermore, a high body mass index (BMI) and an increased amount of visceral fat represent 2 relevant risk factors for the development of $\mathrm{BE}$, irrespective of the presence of GERD [3].

Bariatric surgery proved to be the most effective and long-lasting therapeutic option for morbid obesity and its related diseases [4]. As a consequence the yearly number of surgical procedures progressively increased. In particular in the last decade laparoscopic sleeve gastrectomy (SG) had an exponential growth [5].

Given these data, there is a mounting interest concerning the pathophysiological and clinical effects of bariatric surgery on GERD and its sequelae, aiming to define which surgical procedures, among those ones currently performed, may achieve better outcomes in this particular set of patients.

Laparoscopic Roux-en-Y gastric bypass (LRYGBP) is considered the intervention of choice, either as primary or revision procedure, in morbidly obese patients suffering of GERD with or without HH [6]. To the contrary the indication to SG in patients with GERD is still under debate. A postoperative improvement of reflux symptoms has been reported in some series, while in other series a worsening has been registered [7]. However, in most published studies, GERD diagnosis relies only on clinical symptoms evaluation whereas more objective diagnostic exams such as esophagogastroduodenoscopy (EGD), upper gastrointestinal radiographic swallow (UGI), scintigraphy, manometry metric impedance, or $24 \mathrm{pH}$-manometry are rarely involved [7].

In this paper incidence of GERD in morbidly obese patients before SG and at 58 months' follow-up will be evaluated on the basis of clinical, endoscopic, and histologic data.

\section{Materials and methods}

\section{Study design}

Study design is summarized in Fig. 1. From July 2007 to February 2010, 341 patients referred to our bariatric center for management of their morbid obesity. All patients underwent a multidisciplinary workup including history and physical examination, routine laboratory evaluation, EGD, abdominal ultrasonography, and nutritional and psychiatric evaluation. At EGD, biopsies of the gastroesophageal (GE) junction were routinely taken. UGI was performed only in patients with endoscopic findings such as esophageal diverticula, achalasia-like aspect of the esophagus, and abnormal shape of the stomach and of the duodenum, to rule out possible contraindications to SG.

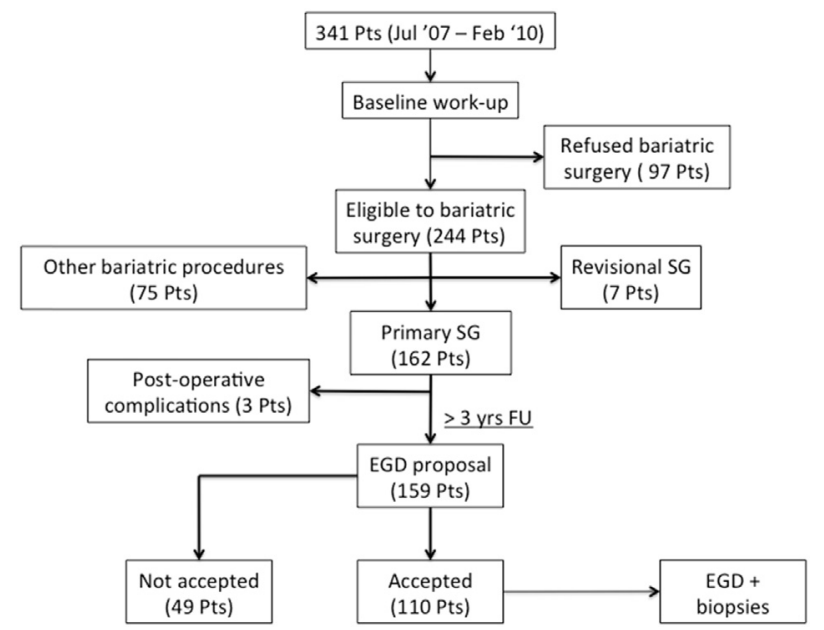

Fig. 1. Study design. $\mathrm{SG}=$ sleeve gastrectomy; $\mathrm{EGD}=$ esophagogastroduodenoscopy.

Additional consultations (e.g., pulmonology, endocrinology) were performed when indicated. GERD symptoms (acid reflux, regurgitation, heartburn) were evaluated by means of the visual analogue scale (VAS). Proton pomp inhibitors (PPI) consumption was registered. All data were collected in a prospective database. Seventy-five patients underwent bariatric procedures other than $\mathrm{SG}$, as for their choice, whereas 97 patients refused bariatric surgery. Seven patients underwent SG as a revision procedure for insufficient weight loss or weight regain after gastric banding (5 patients) or vertical banded gastroplasty (2 patients). In 162 patients primary SG was indicated and performed. After a > 3-year follow-up, from January 2014 to May 2014, a clinical control comprehensive of laboratory tests, VAS evaluation of GERD symptoms, PPI consumption, and EGD (regardless of the presence of GERD symptoms) were prospected to all SG patients.

\section{Endoscopy}

EGD was performed by means of a high-definition gastroscope (Evis Exera II; Olympus Corp., Shinjuku, Tokyo, Japan) with lidocaine spray and under conscious sedation (midazolam iv). In all patients the distance from the upper incisors both to the " $\mathrm{Z}$ " line (squamous-columnar junction) and to the diaphragmatic impression on the esophagus was measured. Esophagitis, when present, was classified according to the Los Angeles Classification [8]. The presence of esophageal biliary-like reflux was registered.

The endoscopic appearance of Barrett's esophagus was evaluated according to the guidelines of the American Gastroenterological Association (AGA) [9] and of the American Society for Gastrointestinal Endoscopy (ASGE) [10]. When BE was suspected, the International C\&M classification (Prague classification) was applied, measuring the circumferential (C) and the maximal proximal extent (M) of the BE mucosal tongue from the upper incisors [11]. 
Table 1

Preoperative versus postoperative GERD symptoms, PPI intake, and endoscopic findings

\begin{tabular}{lllc}
\hline 110 patients & Preoperative & Follow-up & $P$ \\
\hline GERD symptoms & $33.6 \%(37 \mathrm{pts})$ & $68.1 \%(75 \mathrm{pts})$ & $<.0001$ \\
VAS score & 1.8 & 3 & .018 \\
Daily PPI intake & $19.1 \%(21 \mathrm{pts})$ & $57.2 \%(63 \mathrm{pts})$ & $<.0001$ \\
Class A esophagitis & $12.7 \%(14 \mathrm{pts})$ & $46.3 \%(51 \mathrm{pts})$ & $<.0001$ \\
Class B esophagitis & $8.1 \%(9 \mathrm{pts})$ & $32.7 \%(36 \mathrm{pts})$ & $<.0001$ \\
Class C esophagitis & $3.6 \%(4 \mathrm{pts})$ & $11.8 \%(13 \mathrm{pts})$ & .04 \\
Class D esophagitis & 0 & $9.1 \%(10 \mathrm{pts})$ & .0016 \\
Barrett's esophagus & 0 & $17.2 \%(19 \mathrm{pts})$ & $<.0001$ \\
\hline
\end{tabular}

GERD = gastroesophageal reflux disease; PPI = proton pump inhibitors; VAS = visual analogue scale.

In these cases the Seattle biopsy protocol was applied: 4-quadrant biopsy sampling at every 1 to $2 \mathrm{~cm}$ of the columnar lined esophagus [12]. The samples were examined by a pathologist blind to the clinical and endoscopic findings.

\section{SG surgical technique}

Surgical technique has been illustrated elsewhere [13]. SG was tailored on a $48 \mathrm{Fr}$ bougie, and the resection of the stomach started $6 \mathrm{~cm}$ above the pylorus. Staple line was routinely reinforced with an oversewing suture. Special care was taken to completely mobilize the gastric fundus, with meticulous dissection of the posterior gastric wall from the left pillar, to achieve a radical "fundectomy." When $\mathrm{HH}$ was diagnosed, the hiatal area was properly exposed and the defect repaired by posterior hiatoplasty. A gastric remnant of 60-80 mL capacity was obtained. In patients with diagnosis of gallbladder stones, cholecystectomy was performed at the end of the procedure.

\section{Postoperative and follow-up schedule}

An UGI with water-soluble iodinated radiopaque contrast was routinely performed 1 to 3 days after surgery. Patients were discharged on a semisolid diet along with a $40 \mathrm{mg} / \mathrm{d}$ dose of PPI for the first 3 months, followed by $20 \mathrm{mg} / \mathrm{d}$ for the next 3 months. Follow-up schedule entailed medical examination 1 month after surgery and then every 3 months in the first year, every 6 months in the second year, and once a year thereafter. At follow-up, UGI was repeated 1 month after surgery and when deemed necessary thereafter.

\section{Statistical analysis}

Statistical analysis was performed using Stata version 11.2 (Stata Corp., TX, USA). Categorical data were compared using Chi-square; continuous data were compared using $t$ test with CI of 95\%. $P$ values $<.05$ were considered statistically significant.

\section{Results}

SG has been completed laparoscopically in all cases. HH repair (HHR) and cholecystectomy were performed in 16 and 4 patients, respectively (14.5\% and 3.6\%). Of the 162 patients with primary SG, 3 patients $(1.7 \%)$ experienced major postoperative complications (1 leak, 1 bleeding, and 1 dysphagia) and were excluded from the study. A total of 110 SG patients agreed to take part in the study (follow-up rate: $69.1 \%)$ whereas $49(30.9 \%)$ refused. After a mean follow-up of 58 months (range: 55-82), mean BMI decreased from $45.8 \pm 6.3$ to $28.9 \pm 8.7 \mathrm{~kg} / \mathrm{m}^{2}$ with excess weight loss (\%EWL) of $74.1 \pm 21.5 \%$.

Postoperative incidence of GERD symptoms, VAS mean score, and daily intake of PPI were significantly increased compared with the values before LSG (Table 1). At EGD a significant increase in incidence and in severity of erosive esophagitis (EE) was reported, whereas nondysplastic BE was newly diagnosed in 19 patients (17.2\%; Table 1 ). Upward migration of the " $Z$ " line was registered in 81 patients $(73.6 \%)$ with a mean migration length of $3.6 \pm 1.2$ $\mathrm{cm}$ (range: $1-6 \mathrm{~cm}$ ). Incidence and the degree of GERD symptoms did not correlate to the severity of the esophageal lesions, to the presence of BE, and to the extent of " $Z$ " line migration. Patients with Grade C and Grade D esophagitis presented with GERD symptoms only in $33.4 \%$ and $57.2 \%$ of cases, respectively. Furthermore, VAS score was highest in grade A and B esophagitis patients, lower in grade $\mathrm{C}$ and $\mathrm{D}$ esophagitis patients, and lowest in BE patients (Table 2). Biliary-like esophageal reflux was found in 82 patients (74.5\%; (Fig. 2a). In the $16 \mathrm{SG}$ patients with HHR, incidence and severity of GERD symptoms, as well as incidence of BE, were lower, although not significantly so, than in patients who underwent SG alone (Table 3).

\section{Discussion}

Literature data concerning the relationship between GERD and SG are conflicting. Reflux symptoms after this procedure improved in some studies and worsened in others [7]. However, in the Fourth International Consensus Summit on SG in 2012, postoperative GERD was the most frequently reported complication in a collective series

Table 2

Relationship between GERD symptoms and endoscopic findings after SG

\begin{tabular}{|c|c|c|c|}
\hline $\begin{array}{l}\text { Esophagitis degree } \\
\text { (110 pts) }\end{array}$ & $\begin{array}{l}\text { No GERD } \\
\text { symptoms }\end{array}$ & $\begin{array}{l}\text { GERD } \\
\text { symptoms }\end{array}$ & $\begin{array}{l}\text { Mean VAS } \\
\text { score }\end{array}$ \\
\hline Grade A (51 pts) & $27.4 \%$ (14 pts) & $72.6 \%$ (37 pts) & 3.1 \\
\hline Grade B (36 pts) & $25 \%$ (9 pts) & $75 \%$ (27 pts) & 3.4 \\
\hline Grade C (13 pts) & $61.5 \%$ (8 pts) & $38.5 \%$ (5 pts) & 2.8 \\
\hline Grade D (10 pts) & $40 \%$ (4 pts) & $60 \%$ (6 pts) & 2.4 \\
\hline BE (19 pts) & $26.4 \%$ (5 pts) & $73.6 \%$ (14 pts) & 1.3 \\
\hline
\end{tabular}

GERD = gastroesophageal reflux disease; $\mathrm{SG}=$ sleeve gastrectomy; $\mathrm{VAS}=$ visual analogue scale; $\mathrm{BE}=$ Barrett's esophagus. 


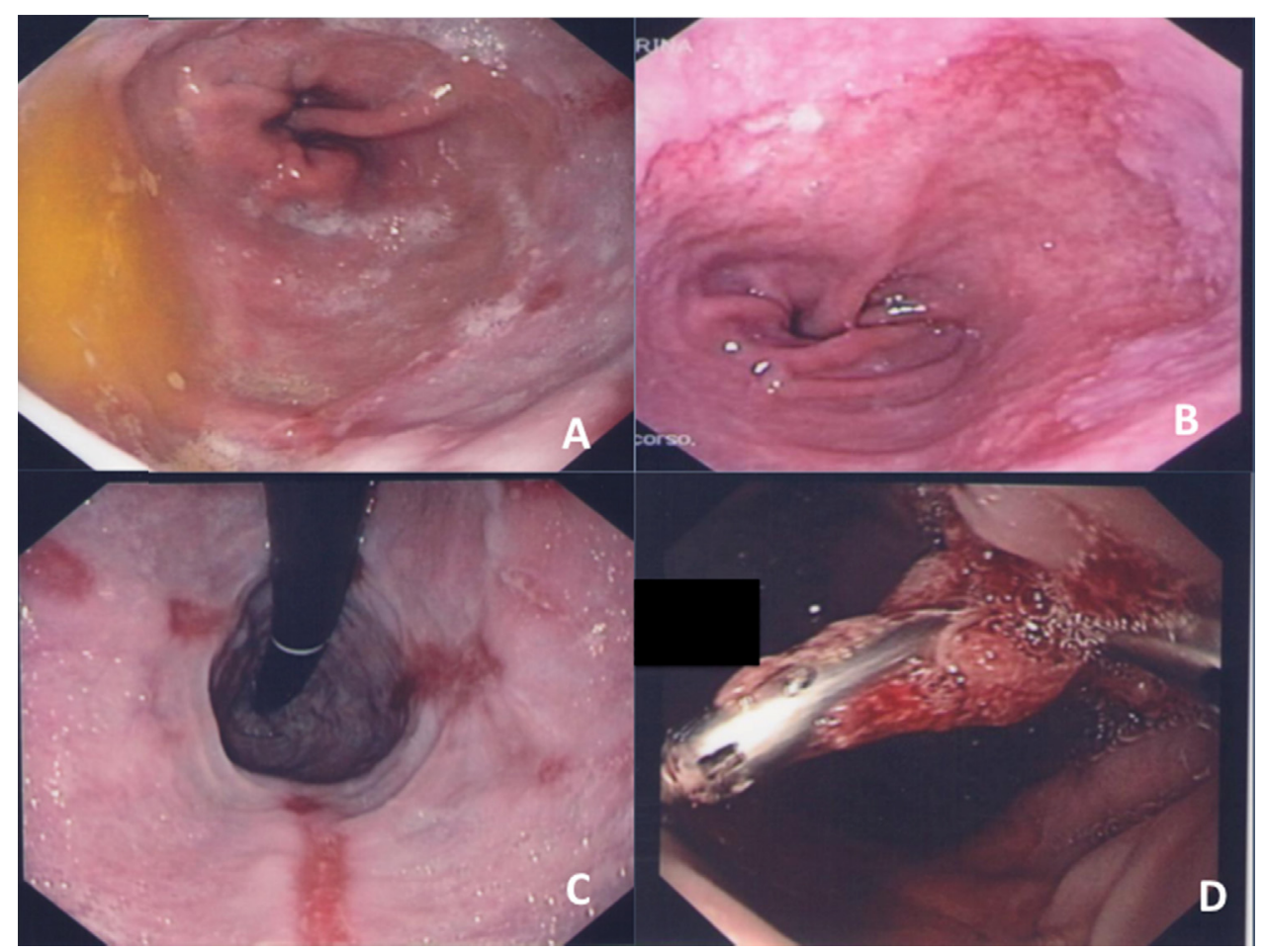

Fig. 2. (A) Biliary-like esophageal reflux; (B) Upward migration of "Z" line; (C) Grade D erosive esophagitis; (D) Hemostatic clip placement after " $Z$ ” line biopsy.

of $>46,000$ SGs performed by 130 surgeons worldwide, with a mean incidence of $7.9 \%$ [14].

Competence of the so-called "antireflux barrier" is based on the proper arrangement of different anatomic structures such as the lower esophageal sphincter (LES), diaphragmatic crus, gastric sling fibers, and the phrenic-esophageal and cardias-phrenic ligaments. The manipulation of any part of this multifactorial barrier, as may occur during SG, can promote or worsen the gastroesophageal reflux.

Table 3

Effect of HHR versus no hiatal repair on GERD symptoms, PPI intake, and endoscopic findings after SG

\begin{tabular}{llll}
\hline & $\begin{array}{l}\text { SG alone } \\
(94 \mathrm{pts})\end{array}$ & $\begin{array}{l}\text { SG }+ \text { HHR } \\
(16 \mathrm{pts})\end{array}$ & $P$ value \\
\hline GERD symptoms & $70.2 \%(66 \mathrm{pts})$ & $56.2 \%(9 \mathrm{pts})$ & .38 \\
Mean VAS score & 3.0 & 2.6 & .60 \\
Daily PPI intake & $56.3 \%(53 \mathrm{pts})$ & $62.5 \%(10 \mathrm{pts})$ & .78 \\
Grade A esophagitis & $50 \%(47 \mathrm{pts})$ & $25 \%(4 \mathrm{pts})$ & .06 \\
Grade B esophagitis & $29.7 \%(28 \mathrm{pts})$ & $50 \%(8 \mathrm{pts})$ & .14 \\
Grade C esophagitis & $10.6 \%(10 \mathrm{pts})$ & $18.7 \%(3 \mathrm{pts})$ & .39 \\
Grade D esophagitis & $8.5 \%(8 \mathrm{pts})$ & $12.5 \%(2 \mathrm{pts})$ & .66 \\
Barrett's esophagus & $19.1 \%(18 \mathrm{pts})$ & $6.2 \%(1 \mathrm{pts})$ & .29 \\
"Z" line upward migration & $3.2 \mathrm{~cm}$ & $2.6 \mathrm{~cm}$ & .93 \\
Biliary-like esophageal reflux & $76.5 \%(72 \mathrm{pts})$ & $62.5 \%(10 \mathrm{pts})$ & .23
\end{tabular}

HHR = hiatal hernia repair; GERD = gastroesophageal reflux disease; PPI = proton pump inhibitors; $\mathrm{SG}=$ sleeve gastrectomy; $\mathrm{VAS}=$ visual analogue scale.
It has been hypothesized that SG determines an imbalance between the intraluminal pressure of the gastric tubule and that of LES. Postoperatively, the former is increased because of lack of compliance to ingested food [15] due to resection of the fundus, the most expandable gastric portion, whereas the latter decreases because of the division of the sling muscle fibers of Helvetius [16]. For this reason, in SG patients, postprandial esophageal regurgitation episodes are significantly increased compared with the preoperative ones, whereas episodes during fasting are not significantly different [17].

Intrathoracic migration of the proximal portion of the gastric tubule may contribute to the decrease of the pressure gradient between the esophago-gastric junction (EGJ) migrated above in the mediastinum (low pressure) and the gastric remnant below in the abdomen (high pressure), favoring GE reflux (Fig. 2b). In the present series, the $\mathrm{Z}$ line migrated upward to an extent in $73.6 \%$ of cases.

An additional confounding factor is the composition of the refluxate. After SG, reflux episodes occur more frequently in the postprandial state and are made up chiefly of nonacid ingested food. This may determine absence of symptoms and relative lack of efficacy of PPI therapy in the management of GERD-related complications [17]. Tai et al., in a series of 47 SG patients, reported that postoperative EE was present in $40.1 \%$ of patients, although they were asymptomatic [18]. Sharma et al. reported a significant improvement of GERD symptoms after SG at a 12-month follow-up, despite worsening of the reflux at 
EGD and at scintigraphy [19]. In our experience, symptomatic GERD occurred more frequently in patients with Grade A and Grade B esophagitis than in patients with more severe (Grade C and Grade D) esophagitis or with BE (Table 2). Furthermore, VAS score was highest in grade A and $\mathrm{B}$ esophagitis patients, lower in grade C and D esophagitis patients, and lowest in BE patients.

At present, most studies investigating the relationship between SG and GERD are based on the clinical evaluation of reflux symptoms. Studies utilizing more objective diagnostic exams, such as EGD, in the pre- and or postoperative period are scarce [7].

EGD data from our series of 110 SG patients studied before and at an average of 58 months after surgery, show that incidence and severity of endoscopic and histologic pathologic findings are significantly higher than those before surgery (Fig. 2c). Severe esophagitis may, at times, complicate EGD mucosal biopsies by determining hemorrhage requiring endoscopic placement of hemostatic clips (Fig. 2d). Most important, incidence and severity of GERD symptoms did not correlate to the endoscopic and histologic findings.

Effect of the HHR on GERD outcome in SG patients is still debated and, at present, there are few published studies dealing specifically with this issue. In the Santonicola et al. study, incidence of GERD symptoms resolution was higher in patients undergoing SG alone than in those with SG plus HHR [20]. To the contrary in the paper by Lyon et al., patients undergoing simultaneous anterior and posterior hiatal repair experienced a noteworthy postoperative improvement of GERD symptoms compared with those who underwent a simple anterior suture of the hiatus or a SG alone [21].

In the present study, in 16 patients with SG plus HHR, incidence and severity of GERD symptoms were slightly lower than that reported in patients with SG alone (Table 3), confirming data previously reported by us [22]. Similarly in the HHR group, incidence of BE, as well as the upward migration of the " $\mathrm{Z}$ " line and the rate of biliary-like esophageal reflux, were lower than in the group of SG alone, although not significantly so (Table 3 ). However, the small number of patients with HHR involved in this study does not allow us to draw firm conclusions.

The high incidence of BE documented in our SG patients is particularly worrisome. Lack of endoscopic controls in the follow-up of SG patients may be detrimental in accurately determine the incidence of GERD-related EE and BE. Results of the present series strongly suggest that careful endoscopic surveillance should be undertaken regardless of absence of reflux symptoms and of PPI intake.

Data from literature concerning the development of $\mathrm{BE}$ after SG are scarce. Braghetto et al. recently reported a $1.2 \%$ incidence (3 cases) of $\mathrm{BE}$ in a cohort of $231 \mathrm{SG}$ patients who did not suffer of reflux symptoms, $\mathrm{HH}$, or BE before surgery [23]. Postoperative "de novo" GERD symptoms and EE developed in $23.2 \%$ and $15.5 \%$ of patients, respectively. BE was diagnosed between 5 and 6 years after surgery, and a laparoscopic conversion to RYGBP was performed [23]. In our series, a conversion to RYGPB was not considered as a first-line treatment after $\mathrm{BE}$ diagnosis, since the yearly progression of nondysplastic $\mathrm{BE}$ to high-grade dysplasia and invasive cancer is .2-.5\% [24]. Moreover, all patients agreed to undergo a long-lasting program of endoscopic surveillance and were compliant with daily assumption of PPIs.

We agree with Gagner who recently postulated that the benefits of SG definitely outweigh its potential and actual risks [25]. In our series, after an average follow-up of 58 months, patients achieved a mean EWL of $74.1 \%$, and, in the vast majority, they were happy with their outcome, confirming what was reported by us in a recent paper [26]. Gagner advocated a protective effect of SG on GERD [25] by mentioning the series by Rebecchi et al., whose patients with preoperative pathologic reflux experienced a significant reduction of GERD symptoms, DeMeester score, and esophageal total acid exposure 24 months after the procedure [27]. Gagner also reported that, despite $>100,000$ cases of biliopancreatic diversion with duodenal switch (BPD-DS, which includes a SG) have been performed over 25 years, no cases of esophageal adenocarcinoma have been described in the literature [25]. Given these data, he assumed that only the preoperative diagnosis of $\mathrm{BE}$, and not the presence of GERD, should be considered a contraindication to SG [25]. However, data from patients with BPD-DS, even if in large cohort, might not be predictive for long-term outcomes of GERD after SG, possibly because in the BPD-DS, the bile juice is diverted from the stomach.

To date, there are no papers investigating, by means of EGD or else, the presence of esophageal biliary reflux after $\mathrm{SG}$, and its actual incidence has not been defined. As indirect evidence, a biliary-like appearance of the esophageal reflux was found in more than two-thirds of the patients in our series (Fig. 2a).

DeMeester et al. postulated that the different components of the refluxed juice contribute differently to the pathogenesis of BE, [28]. Patients with mixed gastric and duodenal reflux had higher incidence and more severe mucosal damage than those with gastric reflux alone [29]. In the duodenal juice, bile salts cause cell damage in a $\mathrm{pH}-$ dependent fashion. At $\mathrm{pH}$ values between 2 and 7, bile salts are soluble and in un-ionized form, cross the mucous cell membranes, and exert a toxic effect on cell mithocondria. Out of this $\mathrm{pH}$ range, bile salts are harmless [30]. Highdosage PPI intake, while easing GERD symptoms, likely does not maintain gastric content $\mathrm{pH}$ consistently above 7 and, consequently, does not prevent mucosal damage. The aforementioned pathophysiological mechanisms may explain findings of the present series.

In the present study a very high incidence of $\mathrm{EE}$ and of $\mathrm{BE}$ in SG patients was observed. These results are in 
contrast with literature data, reporting a significantly lower rate for these complications. Routine use of EGD in the preoperative workup and in the follow-up may possibly explain this discrepancy. Appropriate multicenter and randomized studies are needed to confirm these data.

This study has several flaws, mainly related to the recruitment of patients and the follow-up rate. Nevertheless, it is our opinion that the results observed in our cohort of patients suggest that symptoms evaluation alone could be inadequate to investigate the possible development of GERD and of its related complications after SG.

\section{Conclusion}

Because $\mathrm{SG}$ is performed in an increasing number of patients, postoperative occurrence of $\mathrm{EE}$ and or $\mathrm{BE}$ and its possible long-term sequelae should be carefully considered. Furthermore, it is particularly worrisome that the data of the present study suggest that there is no strict relationship between GERD symptoms and occurrence of EE and or of BE. As a consequence, routine careful endoscopic evaluation in the postoperative surveillance of SG patients should be encouraged, regardless of presence or absence of GERD symptoms. Furthermore, improvements and or modifications of the surgical technique, aimed to reduce esophageal reflux, should be pursued.

\section{Disclosures}

Dr. Alfredo Genco reports that he is Consultant/Trainer of Apollo Endosurgery, Inc., Austin, Texas, since January 2015. The other authors have no commercial associations that might be a conflict of interest in relation to this article.

\section{References}

[1] Hampel H, Abraham NS, El-Serag HB. Meta-analysis: obesity and the risk for gastroesophageal reflux disease and its complications. Ann Intern Med 2005;143(3):199-211.

[2] Eisen GM, Sandler RS, Murray S, Gottfried M. The relationship between gastroesophageal reflux disease and its complications with Barrett's esophagus. Am J Gastroenterol 1997;92(1):27-31.

[3] Edelstein ZR, Farrow DC, Bronner MP, Rosen SN, Vaughan TL. Central adiposity and risk of Barrett's esophagus. Gastroenterology 2007;133(2):403-11.

[4] Sjöström L. Review of the key results from the Swedish Obese Subjects (SOS) trial - a prospective controlled intervention study of bariatric surgery. J Intern Med 2013;273(3):219-34.

[5] Angrisani L, Santonicola A, Iovino P, Formisano G, Buchwald H, Scopinaro N. Bariatric Surgery Worldwide 2013. Obes Surg 2015;25 (10):1822-32.

[6] Frezza EE, Ikramuddin S, Gourash TR, et al. Symptomatic improvement in gastroesophageal reflux disease (GERD) following laparoscopic Roux-en-Y gastric bypass. Surg Endosc 2002;16(7):1027-31.
[7] Chiu S, Birch DW, Shi X, Sharma AM, Karmali S. Effect of sleeve gastrectomy on gastroesophageal reflux disease: a systematic review. Surg Obes Relat Dis 2011;7(4):510-5.

[8] Armstrong D, Bennett JR, Blum AL, et al. The endoscopic assessment of esophagitis: a progress report on observer agreement. Gastroenterology 1996;111(1):85-92.

[9] Spechler SJ, Sharma P, Souza RF, Inadomi JM, Shaheen NJ, American Gastroenterological Association. American Gastroenterological Association medical position statement on the management of Barrett's esophagus. Gastroenterology 2011;140(3):1084-91.

[10] ASGE Standards of Practice Committee. The role of endoscopy in Barrett's esophagus and other premalignant conditions of the esophagus. Gastrointest Endosc 2012;76(6):1087-94.

[11] Sharma P, Dent J, Armstrong D, et al. The development and validation of an endoscopic grading system for Barrett's esophagus: the Prague C \& M criteria. Gastroenterology 2006;131(5):1392-9.

[12] Levine DS, Haggitt RC, Blount PL, Rabinovitch PS, Rusch VW, Reid BJ. An endoscopic biopsy protocol can differentiate high-grade dysplasia from early adenocarcinoma in Barrett's esophagus. Gastroenterology 1993;105(1):40-50.

[13] Basso N, Casella G, Rizzello M, et al. Laparoscopic sleeve gastrectomy as first stage or definitive intent in 300 consecutive cases. Surg Endosc 2011;25(2):444-9.

[14] Gagner M, Deitel M, Erickson AL, Crosby RD. Survey on laparoscopic sleeve gastrectomy (LSG) at the Fourth International Consensus Summit on Sleeve Gastrectomy. Obes Surg 2013;23 (12):2013-7.

[15] Yehoshua RT, Eidelman LA, Stein M, et al. Laparoscopic sleeve gastrectomy-volume and pressure assessment. Obes Surg 2008;18 (9): $1083-8$.

[16] Braghetto I, Lanzarini E, Korn O, Valladares H, Molina JC, Henriquez A. Manometric changes of the lower esophageal sphincter after sleeve gastrectomy in obese patients. Obes Surg 2010;20 (3):357-62.

[17] Del Genio G, Tolone S, Limongelli P, et al. Sleeve gastrectomy and development of "de novo" gastroesophageal reflux. Obes Surg 2014;24(1):71-7.

[18] Tai CM, Huang CK, Lee YC, Chang CY, Lee CT, Lin JT. Increase in gastroesophageal reflux disease symptoms and erosive esophagitis 1 year after laparoscopic sleeve gastrectomy among obese adults. Surg Endosc 2013;27(10):1260-6.

[19] Sharma A, Aggarwal S, Ahuja V, Bal C. Evaluation of gastroesophageal reflux before and after sleeve gastrectomy using symptom scoring, scintigraphy, and endoscopy. Surg Obes Relat Dis 2014;10 (4):600-5.

[20] Santonicola A, Angrisani L, Cutolo P, Formisano G, Iovino P. The effect of laparoscopic sleeve gastrectomy with or without hiatal hernia repair on gastroesophageal reflux disease in obese patients. Surg Obes Relat Dis 2014;10(2):250-5.

[21] Lyon A, Gibson SC, De-loyde K, Martin D. Gastroesophageal reflux in laparoscopic sleeve gastrectomy: hiatal findings and their management influence outcome. Surg Obes Relat Dis 2015;11(3):530-7.

[22] Soricelli E, Iossa A, Casella G, Abbatini F, Calì B, Basso N. Sleeve gastrectomy and crural repair in obese patients with gastroesophageal reflux disease and/or hiatal hernia. Surg Obes Relat Dis 2013;9 (3):356-61.

[23] Braghetto I, Csendes A. Prevalence of Barrett's esophagus in bariatric patients undergoing sleeve gastrectomy. Obes Surg 2016;26 (4):710-4.

[24] Hvid-Jensen F, Pedersen L, Drewes AM, Sørensen HT, Funch-Jensen P. Incidence of adenocarcinoma among patients with Barrett's esophagus. N Engl J Med 2011;365(15):1375-83. 
[25] Gagner M. Is sleeve gastrectomy always an absolute contraindication in patients with Barrett's? Obes Surg 2016;26(4):715-7

[26] Casella G, Soricelli E, Giannotti D, et al. Long-term results after laparoscopic sleeve gastrectomy in a large monocentric series. Surg Obes Relat Dis 2016;12(4):757-62.

[27] Rebecchi F, Allaix ME, Giaccone C, Ugliono E, Scozzari G, Morino M. Gastroesophageal reflux disease and laparoscopic sleeve gastrectomy: a physiopathologic evaluation. Ann Surg 2014;260 (5):909-14.
[28] DeMeester SR, DeMeester TR. The diagnosis and management of Barrett's esophagus. Adv Surg 1999;33:29-68.

[29] Hak NG, Mostafa M, Salah T, et al. Acid and bile reflux in erosive reflux disease, non-erosive reflux disease and Barrett's esophagus. Hepatogastroenterology 2008;55(82-83):442-7.

[30] Kauer WK, Peters JH, DeMeester TR, Ireland AP, Bremner CG, Hagen JA. Mixed reflux of gastric and duodenal juices is more harmful to the esophagus than gastric juice alone. The need for surgical therapy re-emphasized. Ann Surg 1995;222(4):525-31. 